Ann. Zootech., I973, 22 (2), I33-I45.

I.N.R.A.

BIBLIOTHEQUE UO 35906

DOMAINE DE CROUELLE

63039

Clermont-FD Cenex 2

\title{
ENREGISTREMENT GRAPHIQUE DE L'ACTIVITÉ ET DU COMPORTEMENT ALIMENTAIRE DU POULET
}

\author{
J. GOUSSOPOULOS, Y. CARLES, M. PRUD'HON et F. BACOU \\ Station de Physiologie animale, I. N. R. A., \\ École nationale supérieure agronomique, \\ 9, Place Viala, \\ 34060 Montpellier Cedex
}

\section{RÉSUMÉ}

Nous décrivons un dispositif d'enregistrement graphique automatique de l'activité motrice et des consommations d'aliments solide et liquide du Poulet élevé en cage individuelle.

Ce dispositif a été utilisé pour enregistrer ces mesures sur des poulets élevés à la température de $20^{\circ} \mathrm{C} \pm \mathrm{I}^{\circ} \mathrm{C}$, soumis à divers programmes lumineux : lumière de 7 à $2 \mathrm{I}$ heures (I), de 2 à I6 heures (II), lumière permanente (III), obscurité permanente (IV).

Les enregistrements ont été réalisés pendant $2 \mathrm{I}$ jours consécutifs, de l'âge de 5 semaines à l'âge de 8 semaines.

L'activité est répartie au cours de la période d'éclairement exclusivement dans les lots I et II, elle est répartie tout au long des 24 heures dans les lots III et IV.

La consommation d'aliments solides se fait par prises répétées (3o à 55 par jour en moyenne) dont l'amplitude augmente et la fréquence diminue avec l'âge.

Les prises sont réparties de façon régulière pendant toute la période d'activité dans les lots III et IV. Elles ont tendance à être plus fréquentes et plus importantes au cours de l'heure qui suit la reprise d'activité et des trois ou quatre heures qui précèdent l'extinction des lumières dans les lots I et II ; toutefois, au cours de la dernière heure d'éclairement, la consommation s'abaisse, alors que le nombre de repas reste élevé.

Les consommations globales d'aliments, très variables d'un jour à l'autre, et les vitesses de croissance ont été peu différentes d'un lot à l'autre.

En ce qui concerne la boisson, les sujets étudiés ont bu, en moyenne de 22 à 45 fois par jour, les quantités absorbées étant 1,5 à 2 fois supérieures aux quantités d'aliments ingérés; la distribution des prises de boisson était sensiblement la même que celle des repas solides dans chacun des lots.

\section{INTRODUCTION}

Dans des conditions d'alimentation non restrictives, le comportement alimentaire des animaux détermine en partie leur niveau de consommation et par conséquent retentit sur leur croissance. 
Dans le cas du Poulet, pour lequel les études nutritionnelles sont très nombreuses, il existe peu d'études quantitatives précises de comportement alimentaire, en raison sans doute du caractère collectif des conditions d'élevage.

LACASSAGNE et PERo (I954) par enregistrement cinématographique, SiEgei, BEANE et KRAMER (I962) par un procédé photoélectrique, et WEAVER et SIEGEI (I968) par comptage, ont déterminé à heure fixe ou à intervalles réguliers, le nombre d'individus présents à la mangeoire et par conséquent l'évolution dans le temps d'un certain type d'activité alimentaire; FosHEE et al. (I970) se sont basés sur l'enregistrement du niveau sonore émis par des groupes de poulets élevés dans des locaux isolés pour évaluer leur niveau d'activité qui serait étroitement lié à leur comportement alimentaire. D'autres auteurs JENSEN et al. (I962), HASELER (I966) ont étudié la fréquence, la durée et dans certains cas l'importance des prises d'aliments par l'observation d'un petit nombre d'individus au cours d'une période d'éclairement de I2 ou de 24 heures. Ces mesures n'étaient pas répétées quotidiennement, mais espacées en général d'une semaine ce qui ne permet pas, compte tenu de la croissance, d'avoir une idée de l'amplitude des variations journalières. Par ailleurs, cette technique est difficilement applicable pour les sujets placés à l'obscurité.

Il nous a semblé utile de mettre au point un dispositif permettant l'enregistrement graphique du comportement alimentaire et de l'activité du Poulet en croissance élevé en cage individuelle, comme l'ont fait LE MAGNEN et TALLON (Ig66) pour le Rat blanc et Prun'hon et al. (1972) pour le Lapin.

Nous décrivons ici ce dispositif ainsi que les résultats obtenus sur des sujets soumis à différents rythmes d'éclairement - lumière permanente, éclairement alterné I4 L-Io $\mathrm{D}$, obscurité permanente.

\section{MATÉRIEL ETT MÉTHODES}

\section{Dispositif d'enregistrement}

\section{Activité.}

Deux cages mesurant $50 \times 80 \times 55 \mathrm{~cm}$ ont été munies d'un plancher grillagé susceptible de bouger au moindre mouvement de l'animal ; à cet effet, il est suspendu par 4 fils métalliques (multibrins 20/10 $\mathrm{mm}$ ), un système de butées permettant de régler l'amplitude des mouvements du plancher. Celui-ci est directement relié par un fil de lin au stylet inscripteur d'un kymographe (Braun Aparatebau, type 1o7o). Le déroulement du papier est continu, la vitesse de défilement étant de $2,25 \mathrm{~cm} /$ heure.

\section{Consommation.}

Elle est contrôlée sur un animal seulement.

La mangeoire de la cage est fixée sur l'un des plateaux d'une balance automatique, une tare faisant équilibre ; ce plateau est relié à un second stylet inscripteur du même kymographe, les mouvements étant amplifiés 5 fois par un système de leviers à bras inégaux.

L'eau est distribuée dans un abreuvoir automatique alimenté par un tube souple relié à une éprouvette servant de réservoir. Un flotteur qui suit le niveau d'eau est directement relié à un troisième stylet inscripteur du kymographe. Enfin, un quatrième stylet inscrit un trait continu pendant toutes les périodes d'éclairement.

Nous donnons sur la figure $I$, un schéma du dispositif expérimental. Sur la figure 2 sont représentés les enregistrements d'activité, et de consommation d'aliments solide et liquide d'un coquelet. 


\section{Conditions expérimentales.}

Le dispositif expérimental est placé dans un local sans fenêtre où l'on peut faire varier la température et la durée d'éclairement. Dans ces essais préliminaires, la température a été maintenue à $20^{\circ} \mathrm{C} \pm \mathrm{I}^{\circ} \mathrm{C}$, l'intensité d'éclairement était d'environ $\mathrm{I} 200$ lux.

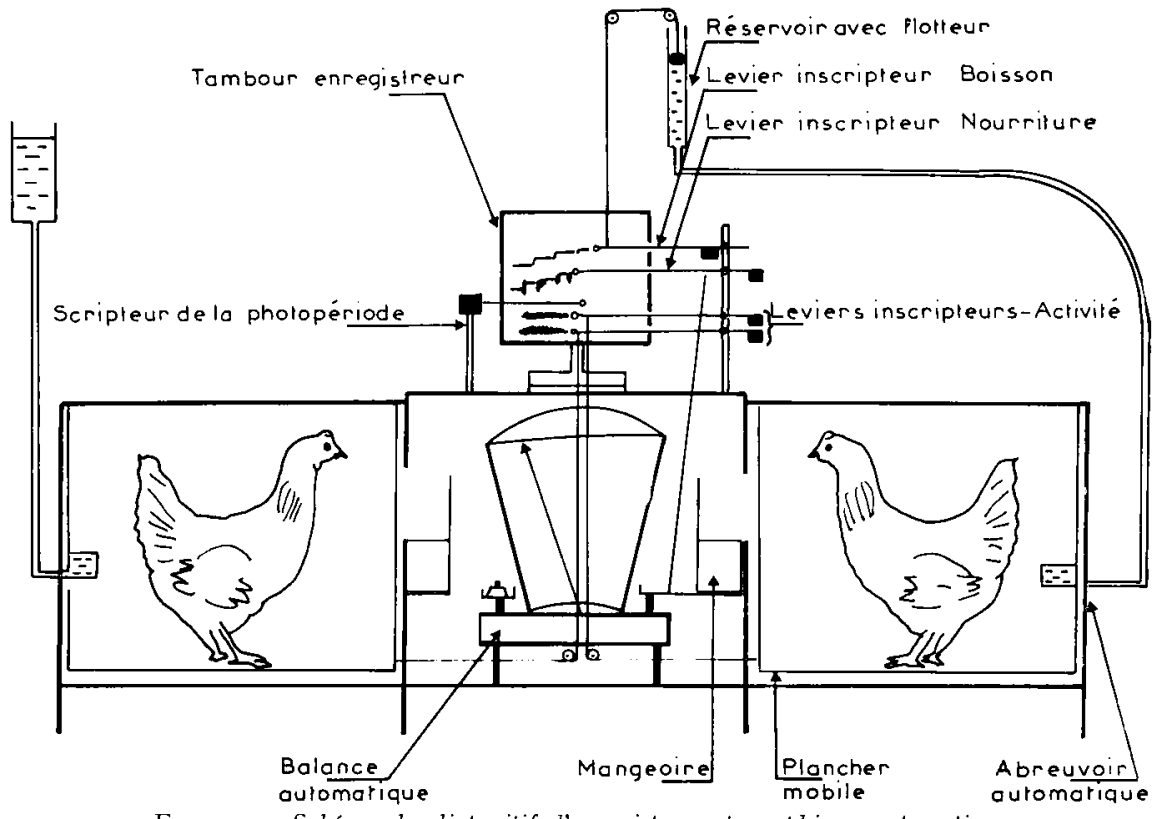

FIG. I. - Schéma du dispositif d'enregistrement graphique automatique

des prises d'aliments solide et liquide d'un poulet et de l'activité motrice de deux poulets

4 types d'éclairement ont été testés sur des groupes de 3 animaux en croissance entre la cinquième et la huitième semaine:

I animal témoin en cage à fond immobile,

I animal dont on contrôle l'activité,

I animal dont on contrôle l'activité et la consommation.

Les différents éclairements étaient les suivants :

- Groupc I : $\mathrm{L}=\mathrm{I} 4, \mathrm{D}=\mathrm{IO}$, lumière de 7 à $2 \mathrm{I}$ heures

- Groupe II : $\mathrm{L}=\mathrm{I} 4, \mathrm{D}=\mathrm{I0}$, lumière de 2 à $\mathrm{I} 6$ heures

- Groupe III : LL = lumière permanente

- Groupe IV : DD = obscurité permanente

Les anima ux du groupe I avaient la durée et la répartition d'éclairement couramment utilisée dans notre élevage; le groupe II avait la même durée d'éclairement mais avec un décalage de 5 heures dans le déclenchement et l'extinction de la lumière de manière à ce que les effets accidentels d'environnement - reprise de travail du personnel, distribution d'aliments, bruits divers s'il y en a - ne puissent être confondus avec les effets des variations d'éclairement; dans ces deux groupes, les animaux préalablement soumis à un éclairement permanent depuis leur naissance ont été placés en régime alterné à l'âge de 3 semaines, soit 2 semaines avant le début des enregistrements.

Les animaux du groupe III ont été maintenus à la lumière permanente depuis leur naissance.

Enfin, les animaux du groupe IV ont été placés à l'obscurité totale, après une courte période de 3 jours où ils ont reçu un éclairement extrêmement faible - de l'orclre de I lux - leur permettant de repérer l'emplacement de la mangeoire et de l'abreuvoir.

Pendant la durée de ces essais, les foulets ont reçu à volonté de l'cau ainsi qu'vrc farine alimentaire de croissance. 







\section{RÉSULTATS ET DISCUSSION}

Les poids moyens à $5,6,7$ et 8 semaines étaient les suivants :

$\begin{array}{rcccc}\text { Groupe } & 5 \mathrm{sem} & 6 \mathrm{sem} & 7 \mathrm{sem} . & 8 \mathrm{sem} . \\ -\mathrm{I} & - & - & - & - \\ \text { II } & 540 & 730 & 950 & \text { I 200 } \\ \text { III } & 560 & 730 & 890 & \text { I I60 } \\ \text { IV } & 590 & 780 & 990 & \text { I 220 }\end{array}$

La vitesse de croissance moyenne a peu différé d'un lot à l'autre ; les valeurs extrêmes : $3 \mathrm{I}, 4$ et $28,6 \mathrm{~g} / \mathrm{j}$ étaient enregistrées dans les lots I et II soumis pourtant aux mêmes durées d'éclairement. L,es animaux témoins (plancher fixe) avaient la même croissance que les animaux soumis au contrôle d'activité (plancher mobile).

La plupart des auteurs constatent une croissance plus rapide des poulets éclairés de façon permanente (SHUTZE et al., Ig60; BEANE et al., I962 et I965; WEAVER et SIEGEL, I968). Le faible nombre de sujets contrôlés ne nous a pas permis de vérifier ce point.

\section{A. - Activité}

Sur la figure 3, sont représentés, juxtaposés, les enregistrements d'activité des animaux des différents groupes au cours de 2 I jours consécutifs.

Les animaux élevés en lumière et obscurité alternées ont une activité relativement constante pendant la photopériode claire, elle cesse presque complètement pendant la période d'obscurité.

Dans les deux autres groupes, par contre, l'activité se répartit sur l'ensemble des 24 heures avec, peut être, de légères variations d'intensité que nous nous proposons d'étudier à l'aide de techniques d'analyses plus fines (analyse spectrale, cosinor...)

\section{B. - Alimentation solide}

La consommation d'aliments solides, présentés sous forme pulvérulente, s'effectue au cours de repas bien individualisés dont nous avons pu mesurer le nombre, l'importance, la durée totale et la répartition temporelle.

I.e nombre de repas (tab1. I) est de l'ordre de 30 à 55 par jour et semble varier assez sensiblement d'un individu à l'autre et d'un jour à l'autre chez le même individu; il aurait, en outre, tendance à diminuer avec l'âge au cours des 3 semaines de croissance contrôlées.

Ces résultats concordent avec ceux obtenus par JENSEN et al. (I962) et HASELER (I966). Par ailleurs, LACASSAGne et Pero (I954), WEAVER et SiEgei (I968) ont signalé une diminution avec l'âge du pourcentage d'animaux présents à la mangeoire au cours de leurs observations périodiques.

La quantité journalière d'aliments consommés, dont les variations interindivi- 


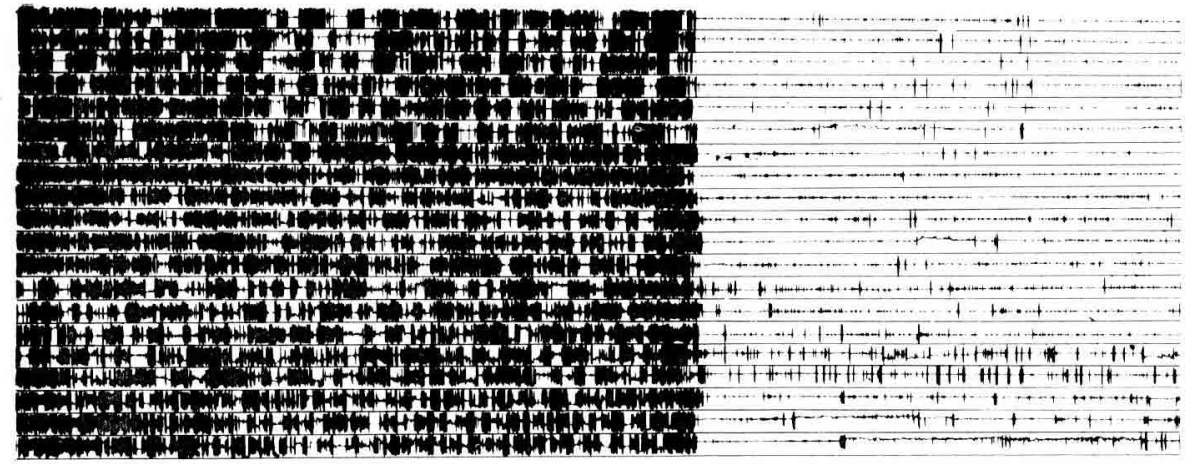

(1)
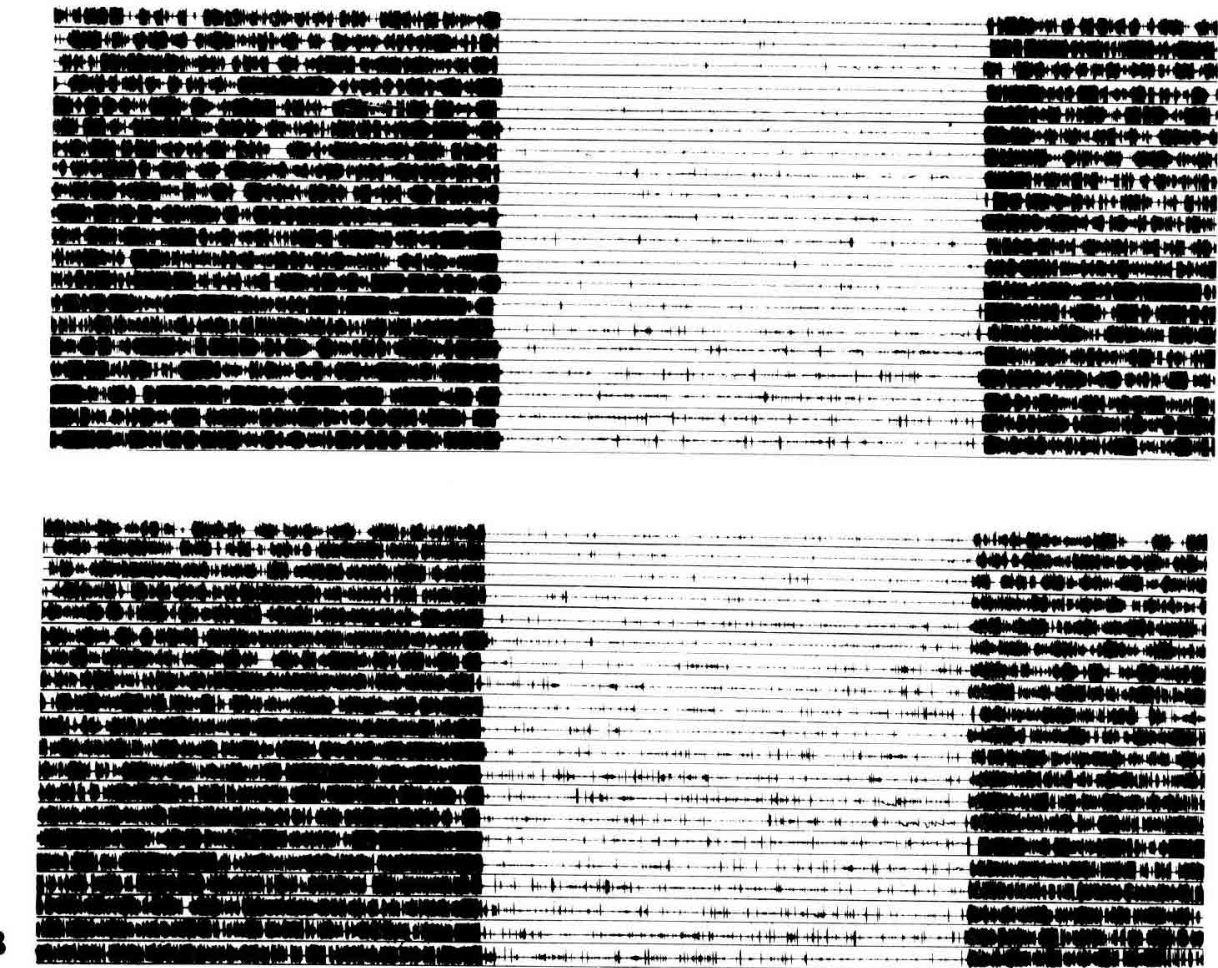

FIG. 3. - Envegistrement de l'activité motrice de quatre groupes de deux poulets soumis

A Lot I I4 L-Io D (lumière de 7 heures à 2 I heures)

$\mathrm{B}$ Lot II I4 L-Io D (lumière de 2 heures à I 6 heures)

Chaque ligne représente une journée d'enregistremant d'activité, les 


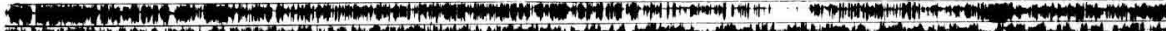

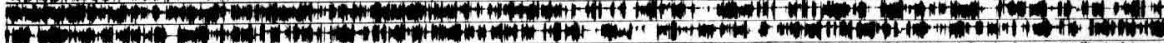

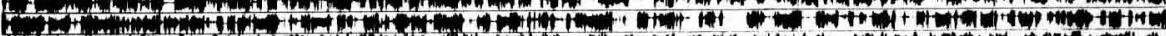
A



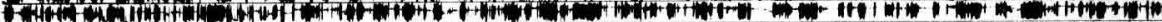

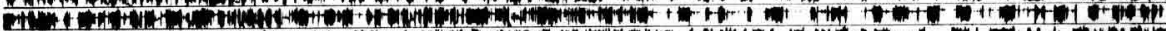


\&

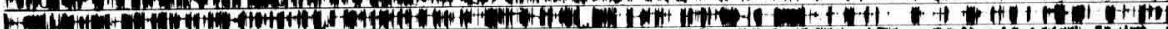
-

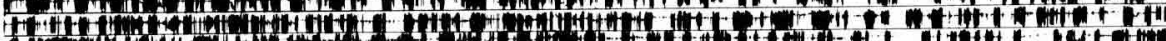

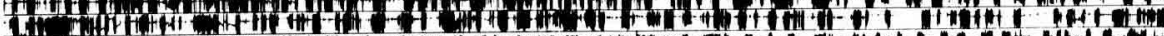
1 int



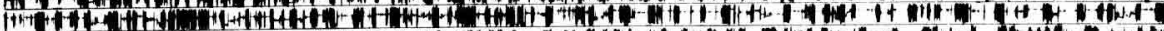

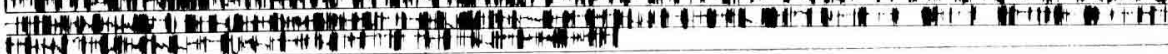

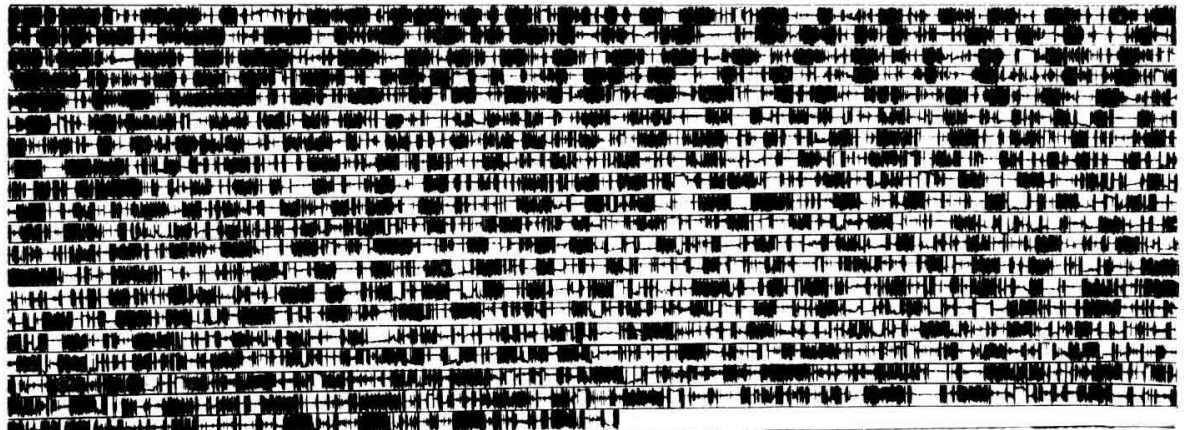

C FP

A OH

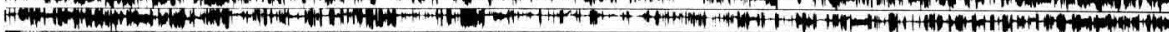

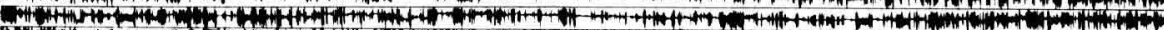

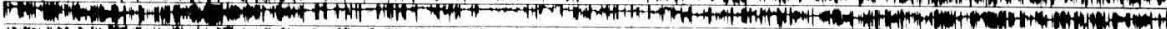



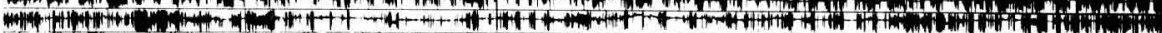
Font

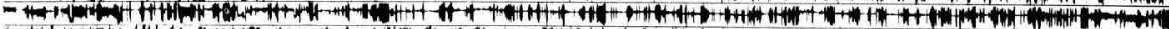

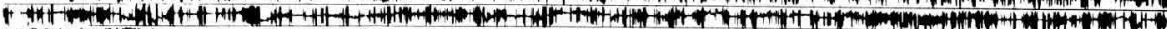

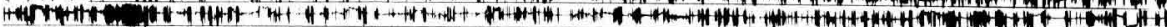
Ho

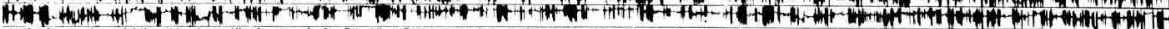

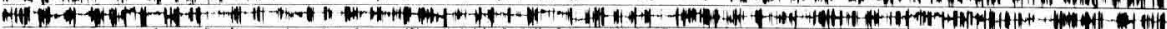

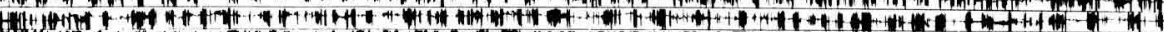

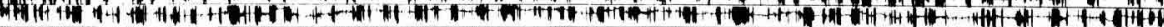

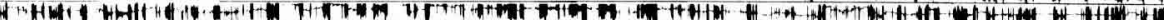

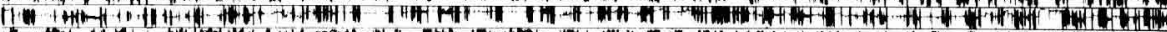

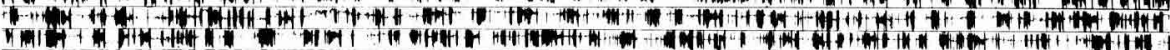

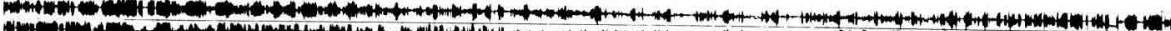
W

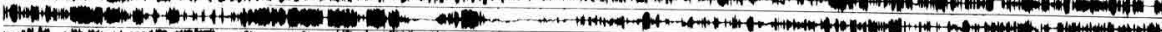

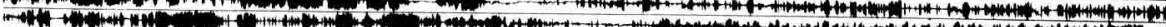

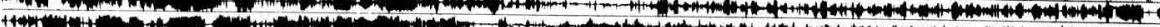

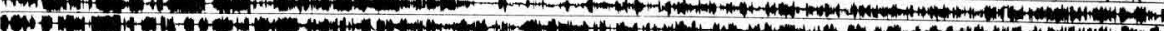
M.

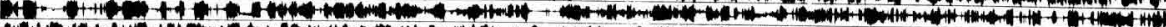

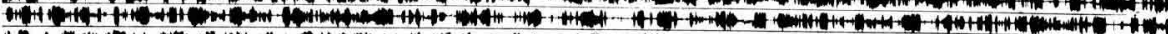

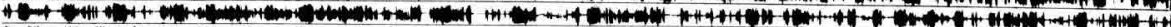

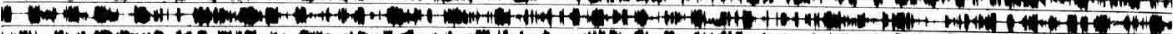

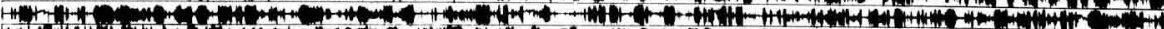

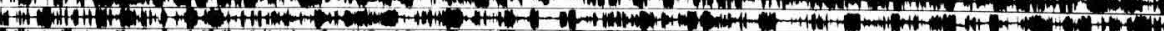



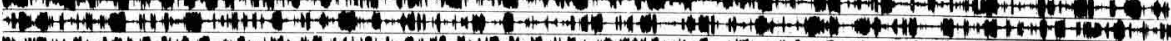
5.

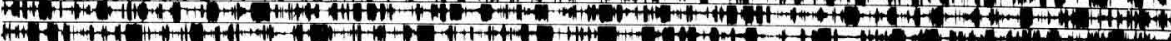



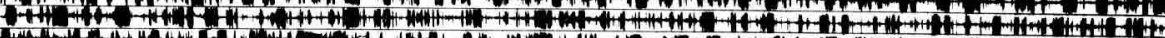

D

$\dot{a}$ des types diffêrents d'éclairement au cours d'une période de 21 jours consécutifs

C Lot III LL Iumière permanente

D Lot IV DI) obscurité permanente

2 I jours ont été superposés. Pour chaque lot on a utilisé deux poulets 
duelles et intra-individuelles sont moins marquées, augmente avec l'âge. Il en est de même a fortiori, de l'importance unitaire des repas qui passe en moyenne de 2 $\mathrm{à}^{\top} \mathrm{g}$ entre la cinquième et la huitième semaine.

TABLEAU I

Fréquence journalière des repas et quantité consommée

\begin{tabular}{|c|c|c|c|c|c|c|c|}
\hline \multirow{2}{*}{ No des lots } & \multirow{2}{*}{ Photopériode } & \multicolumn{3}{|c|}{ Nombre journalier de repas } & \multicolumn{3}{|c|}{ Quantité journalière consommée } \\
\hline & & $5-6$ sem. & 6-7 sem. & $7-8$ sem. & 5-6 sem. & 6-7 sem. & $7-8$ sem. \\
\hline I & L $14 \quad \mathrm{D} 10$ & $34 \pm 7,7 *$ & $34 \pm 7,7$ & $31 \pm 7,1$ & $102 \pm 7,1$ & $110 \pm 8,9$ & $123 \pm 4,5$ \\
\hline II & L 14 D 10 & $54 \pm 13,4$ & $51 \pm 8,1$ & $35 \pm 3,7$ & $76 \pm 8,9$ & $105 \pm 8,1$ & $121 \pm 3,7$ \\
\hline III & LL & $49 \pm 8,4$ & $32 \pm 5,3$ & $32 \pm 1,7$ & $91 \pm 4,6$ & $112 \pm 6,9$ & $119 \pm 6,2$ \\
\hline IV & $\mathrm{DD}$ & $53 \pm 7,7$ & $32 \pm 5,5$ & $28 \pm 3,0$ & $91 \pm 16,9$ & $114 \pm 8,0$ & $138 \pm 9,8$ \\
\hline
\end{tabular}

* Écart type.

TABLEAU 2

Durée journaliève de présence à la mangeoire et intervalle moyen entre deux repas

\begin{tabular}{|c|c|c|c|c|c|}
\hline \multirow{2}{*}{ No des lots } & \multirow{2}{*}{ Photopériode } & \multicolumn{3}{|c|}{ Durée de présence } & \multirow{2}{*}{$\begin{array}{c}\text { Intervalle moyes } \\
\text { entre } 2 \text { repas }\end{array}$} \\
\hline & & 5-6 sem. & 6-7 sem. & $7-8$ sem. & \\
\hline I & L $14 \quad$ D 10 & 3 h $55 \pm 30^{\prime *}$ & $4 \mathrm{~h} 50 \pm 21^{\prime}$ & $3 \mathrm{~h} 40 \pm 10^{\prime}$ & $18 \mathrm{mn}$ \\
\hline II & L $14 \quad$ D 10 & $4 \mathrm{~h} 45 \pm 24^{\prime} *$ & $4 \mathrm{~h} 15 \pm 14^{\prime}$ & $4 \mathrm{ho0} \pm 16^{\prime}$ & $13 \mathrm{mn}$ \\
\hline III & LL & $7 \mathrm{~h} 30 \pm 70^{\prime}$ & $6 \mathrm{~h} 40 \pm 67^{\prime}$ & $6 \mathrm{~h} 00 \pm 33^{\prime}$ & $32 \mathrm{mn}$ \\
\hline IV & $\mathrm{DD}$ & $4 \mathrm{~h} 15 \pm 36^{\prime}$ & 3 h $45 \pm 25^{\prime}$ & $3 \mathrm{~h} 20 \pm 24^{\prime}$ & $32 \mathrm{mn}$ \\
\hline
\end{tabular}

* Écart type.

La durée totale de présence à la mangeoire (tabl. 2), obtenue en faisant la somme des curées unitaires des repas au cours des 24 heures, est de l'ordre de 4 heures chez les individus des groupes I, II et IV et de $6 \mathrm{~h} 30 \mathrm{chez}$ l'individu du groupe III. En l'absence de données plus nombreuses on ne peut dire s'il s'agit d'un phénomène dû à l'éclairement ou à une variation individuelle. Ces résultats sont assez voisins 
咅

$3 \% 8$

s.s.

$\ldots$

628

$\underline{s . \underline{s . s}}$

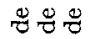

!!!
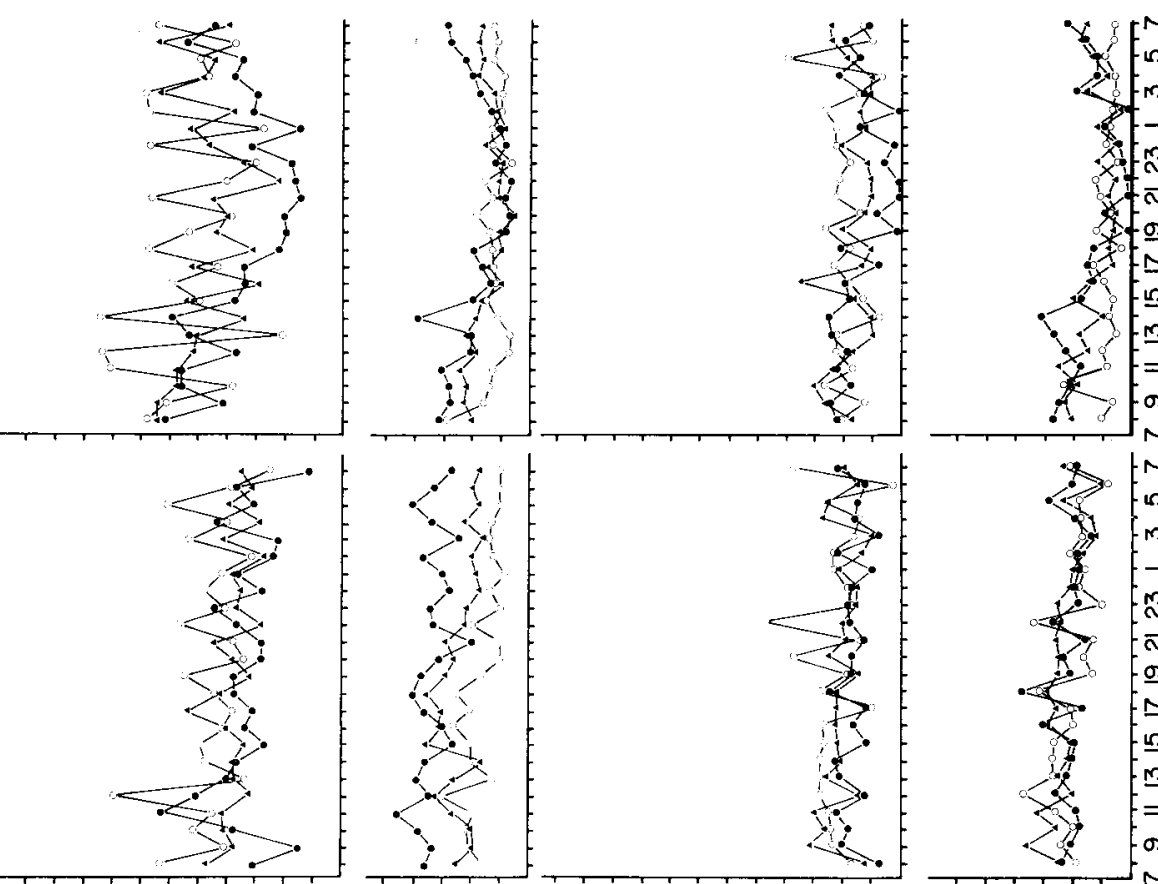

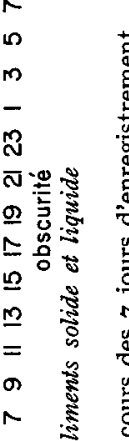
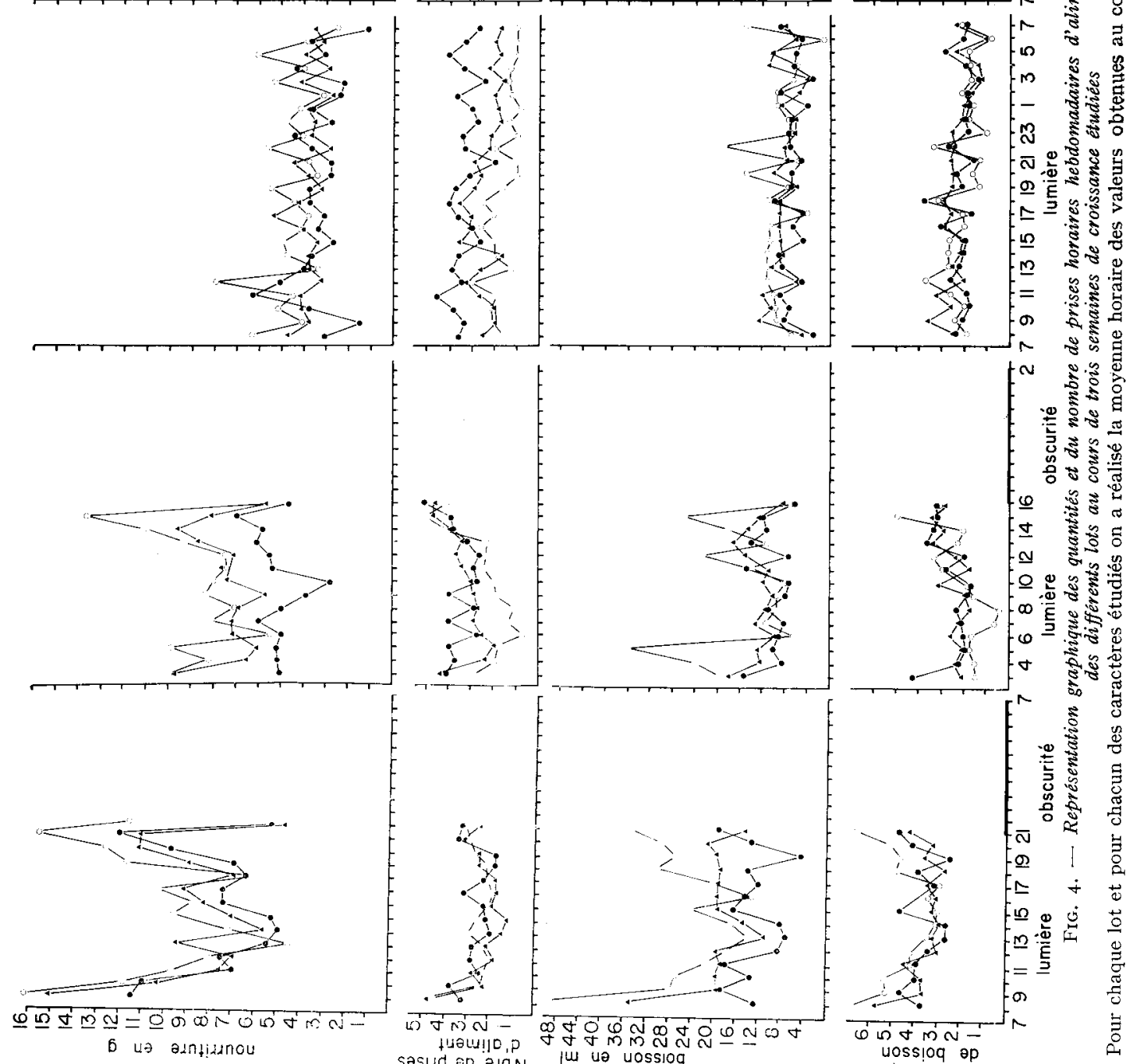

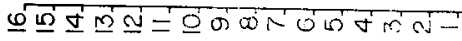

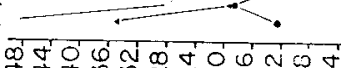
iu ve uossiog




de ceux obtenus par HASELER (I966), compte tenu des variations individuelles importantes qu'il signale; ils sont, par contre, sensiblement supérieurs aux résultats enregistrés par JENSEN et al. (Ig62) sur des poussins plus jeunes consommant également de la farine et soumis à un éclairement alterné de I2 I,-I2 D. I a durée totale de présence à la mangeoire semble relativement constante chez les poulets en rythme I4 $\mathrm{L}_{\text {-IO }} \mathrm{D}$, elle a plutôt tendance à diminuer avec l'âge chez les sujets placés en obscurité ou en lumière permanente. Dans ce cas, les repas sont répartis tout au long des 24 heures; l'intervalle moyen entre deux repas successifs étant environ deux fois plus élevé qu'avec le rythme I4 L-Io D ; autrement dit, il semble qu'à un âge donné, le Poulet consomme une quantité à peu près constante d'aliments, indépendante du rythme d'éclairement dont dépendrait étroitement au contraire, le rythme des repas. JENSEN et al. (I962) soulignent également cette constance de consommation lorsque l'aliment est présenté sous forme de granulé ou de farine, la granulation n'ayant pour effet que de diminuer le temps consacré au repas.

Sur la figure 4 sont représentées, semaine par semaine et heure par heure, les fréquences moyennes des repas et les quantités moyennes d'aliments consommées.

Les animaux recevant un éclairement alterné (groupes I et II) mangent uniquement au cours de la période claire et ont tendance à manger et à boire plus souvent et davantage au révail ainsi qu'au cours des 3 ou 4 heures précédant l'extinction de la lumière ; toutrfois, si la fréquence des repas reste élevée au cours de la derrière heure d'éclairement, les quantités consommées sont sensiblement plus faibles.

L'activité qui en résulte présente donc deux pics bien marqués qui ont été analysés par BACOU (I970) pour ce qui concerne ces animaux. De nombreux auteurs, d'ailleurs (cf. revue d'Aschoff, I966), ont mis en évidence l'existence de deux pics d'activité situés à l'aube et au crépuscule dans diverses espèces animales. En ce qui concerne le Poulet, nos résultats sont en accord avec ceux de SIEGEI, et al. (I962), Weaver et Sieger (I968), Centa, Foshee er Howes (I969) qui signalent, chez des sujets soumis à un rythme alterné d'éclairemcnt, une activité ou une présence à la mangeoire accrue au moment de l'allumage et en anticipation de l'extinction des lumières.

En lumière et en obscurité permanente, la fréquerce des repas et la quantité consomnéa semblent réparties de façon régulière tout au long de la journée, avec toutefois, des variations d'amplitude qui, chez le sujet vivant à l'obscurité, ne paraissent pas distribuées de façon aléatoire.

\section{C. - Alimentation liquide}

La fréquence et l'importance quotidienne de la consommation d'eau sont rapportées au tableau 3. Les animaux étudiés ont bu, en moyenne de 22 à 45 fois par jour; les quantités quotidiennes consommées sont $\mathrm{I}, 5$ à 2 fois plus élevées que les quantités d'aliment ingérées, la proportion restant constante pour un individu donné.

L'évolution de la fréquence des prises et de l'importance des consommations pendant les trois semaines de croissance étudiées est très analogue à celle des repas solides.

Les prises de boisson sont réparties tout au long de la période claire dans les lots I, II et III et de la période obscure dans le lot IV ; leur fréquence et les quantités 
absorbées étant toutefois plus élevées à l'approche de l'extinction de la lumière dans les lots I et II.

Enfin, nous n'avons constaté aucune relation immédiate dans la succession des repas solide et liquide ni dans leur importance respective.

\section{TABLEAU 3}

Fréquence journalière des prises de boisson et quantité consommée

\begin{tabular}{|c|c|c|c|c|c|c|c|}
\hline \multirow{2}{*}{ No des lots } & \multirow{2}{*}{ Photopériode } & \multicolumn{3}{|c|}{$\begin{array}{l}\text { Nombre journalier } \\
\text { de prises de boisson }\end{array}$} & \multicolumn{3}{|c|}{ Quantité journalière consommée } \\
\hline & & 5-6 sem. & 6-7 sem. & $7-8$ sem. & 5-6 sem. & 6.7 sem. & 7-8 sem. \\
\hline I & L $14 \quad$ D 10 & $41 \pm 5,6^{*}$ & $43 \pm 8,7$ & $45 \pm 2,5$ & $185 \pm 16$ & $195 \pm 20$ & $268 \pm 12$ \\
\hline II & L $14 \quad$ D 10 & $35 \pm 6,0$ & $32 \pm 7,2$ & $24 \pm 5,3$ & $133 \pm 27$ & $180 \pm 12$ & $190 \pm 11$ \\
\hline III & LL & $37 \pm 3,7$ & $32 \pm 5,9$ & $36 \pm 7,4$ & $198 \pm 5$ & $222 \pm 12$ & $230 \pm 14$ \\
\hline IV & $\mathrm{DD}$ & $31 \pm 7,2$ & $26 \pm 7,0$ & $22=2,7$ & $140 \pm 14$ & $175 \pm 13$ & $188 \pm 17$ \\
\hline
\end{tabular}

* Écart type.

\section{CONCLUSION}

Nous avons décrit un dispositif expérimental permettant un enregistrement graphique sensible et précis de l'activité motrice et du comportement alimentaire de poulets en croissance élevés en cages individuelles.

Ce dispositif devrait permettre d'étudier l'influence de divers paramètres nature de l'alimentation, rythme d'éclairement, etc. - sur les caractéristiques de la consommation d'eau et de matière sèche indépendamment des phénomènes de concurrence entre individus dont on sait (Tolman, I964, I965, I968; TolmaN et WILSON, I965; SHRECK et al., I968) qu'ils peuvent avoir une incidence sur le comportement alimentaire de poulets élevés en groupes.

Les résultats enregistrés, bien que peu nombreux, ont mis en évidence l'importance des effets de la lumière sur le comportement alimentaire du poulet ainsi que ses facultés d'adaptation à des rythmes extrêmes.

Ils ont permis, dans le cas d'éclairements alternés, de confirmer l'existence d'une consommation accrue d'aliments préludant à l'extinction de la lumière assez comparable au mécanisme de consommation prévisionnelle décrit chez le Rat blanc par LE Magnen et TAlion (Ig66). 


\section{SUMMARY}

\section{AUTOMATIC GRAPH RECORDING OF MOVING ACTIVITY AND FEEDING BEHAVIOUR IN THE CHICKEN}

A device for automatic graph recording of the moving activity as well as the liquid and solid feed intake of individually caged chickens is described.

The chickens were kept at a temperature of $20^{\circ} \mathrm{C} \pm \mathrm{I}^{\circ} \mathrm{C}$ and subjected to various lightening programmes : light from 7 a. m. till 9 p. m. (I), from 2 a. m. till 4 p. m. (II), permanent light (III) permanent darkness (IV).

Recordings were made for $2 \mathrm{I}$ consecutive days from the age of 5 weeks to the age of 8 weeks.

In groups I and II, moving activity was only recorded during the period of light whereas it was distributed over 24 hours in groups III and IV.

The solid feed intake was repeated, on an average, 30 to 55 times per day. The amounts of feed eaten per meal increased with age whereas the number of meals decreased.

In groups III and IV the feed intakes were regularly distributed over the whole period of activity. They tended to be more frequent and larger during the hour following the renewal of activity as well as during the three or four hours preceding the suppression of light in groups I and II. However, during the last hour of lightening, feed intake decreased, whereas the number of meals remained high.

There was small differences from one group to another as regards the growth rate and the total feed intake, which varied much from one day to another.

With respect to water consumption, the animals drank, on an average, 22 to 45 times per day; the amounts were I.5 to 2 times higher than the solid feed intake. The distribution of water intakes was almost the same as that of solid feed intakes in each of the groups studied.

\section{RÉFÉRENCES BIBLIOGRAPHIQUES}

Aschoff J., I966. Circadian activity pattern with two peaks. Ecology. 47, 657-662.

Bacou F., I970. Rythme circadien de l'activité motrice chez le Poulet et le Lapin domestique. Diplôme d'Études approfondies, 30 p., Montpellier.

Beane W. L., Siegel P. B., Siegel H. S., I962. The effects of light on body weight and feed conversion of broilers. Poult. Sci., 41, I350-I35I.

Beane W. L., Siegel P. B., Siegel H. S., I965. Light environment as a factor in growth and feed efficiency of meat type chickens. Poult. Sci, 44, I0o9-IOI2.

Centa D. M., Foshee D. P., Howes J. R., I969. Control of feeding behaviour in poultry. Physiological Reports, 24, I53-I54.

Foshee D. P., Centa D. M., McDaniel G. R., Rollo C. A., 197o. Diurnal activity patterns of broilers in a controlled environment. Poult. Sci., 49, I5I4-I5I7.

Haseler F., 1966. Beobachtungen über lireb - und Ruhezeiten bei Broilerküken von der I. bis ro. Lebenswoche. Arch. Geflügelz. Kleintierk., 15, 287-315.

Jensen L. S., Merrill L. H., Reddy C. V., McGinnis J., I962. Observations on eating patterns and rate of food passage of birds fed pelleted and unpelleted diet. Poult. Sci., 41. I4I4-I4I9.

Lacassagne L., Pero R., i954. Étude cinématographique du Poussin de o à ro semaines. Proc. $X X t h$ world's Poult. Congr., Edinburgh, 353-358.

Le Magnen J., Tallon S., ig66. La périodicité spontanée de la prise d'aliments ad libitum du Rat blanc. J. Physiol., Paris, 58, 56r-564.

Prud'hon M., Carles Y., Goussopoulos J., Koehl P. F., I972. Enregistrement graphique des consommations d'aliments solide et liquide du Lapin domestique nourri ad libitum. Ann. Zootech. 21, $45 \mathrm{I}-460$.

Shreck P. K., Sterritt G. M., Smith M. P., Stilson D. W., I968. Environmental factors in the development of eating in chicks. Anim. Behav., 11, 306-309.

Shutze J. V., Jensen L.S., Carver J.S., Matson W. E., Ig60. Influence of various lighting regimes on the performance of growing chickens. Tech. bull. Washington agric. Exp. stn. $\mathrm{n}^{\circ} 36$.

Siegel B. P., Beane W. L., Kramer C. Y., ig6z The mesurement of feeding activity in chickens to 8 weeks of are. Poult. Sci, 41, I4I9-1422. 
Tolman C. W., I964. Social facilitation of feeding behaviour in the domestic chick. Anim. Behav., 12, $245-25$ I.

Tolman C. W., 1965. Emotional behaviour and social facilitation of feeding in domestic chicks. Anim Behav., 13, 493-496.

Tolman C. W., I968. The varieties of social stimulation in the feeding behaviour of domestic chicks. Anim. Behav., 30, 275-286.

Tolman C. W., Wilson G. F., 1965. Social feeding in domestic chicks. Anim. Behav., 13, 134-I42. Weaver W. D. Jr, Siegel P. B., I968. Photoperiodism as a factor in feeding rhythms of broiler chickens. Poult. Sci., 47, II48-II54. 